\title{
Monitoring of anesthetic depth and EEG band power using phase lag entropy during propofol anesthesia
}

\author{
Hye Won Shin ${ }^{1 *} \mathbb{D}$, Hyun Jung Kim², Yoo Kyung Jang ${ }^{1}$, Hae Sun You', Hyub Huh', Yoon Ji Choi ${ }^{3}$, \\ Seung Uk Choi ${ }^{1}$ and Ji Su Hong ${ }^{1}$
}

\begin{abstract}
Background: Phase lag entropy (PLE) is a novel anesthetic depth indicator that uses four-channel electroencephalography (EEG) to measure the temporal pattern diversity in the phase relationship of frequency signals in the brain. The purpose of the study was to evaluate the anesthetic depth monitoring using PLE and to evaluate the correlation between PLE and bispectral index (BIS) values during propofol anesthesia.
\end{abstract}

Methods: In thirty-five adult patients undergoing elective surgery, anesthesia was induced with propofol using target-controlled infusion (the Schneider model). We recorded the PLE value, raw EEG, BIS value, and hemodynamic data when the target effect-site concentration (Ce) of propofol reached 2, 3, 4, 5, and $6 \mu \mathrm{g} / \mathrm{ml}$ before intubation and $6,5,4,3,2 \mu \mathrm{g} / \mathrm{ml}$ after intubation and injection of muscle relaxant. We analyzed whether PLE and raw EEG data from the PLE monitor reflected the anesthetic depth as the Ce of propofol changed, and whether PLE values were comparable to BIS values.

Results: PLE values were inversely correlated to changes in propofol Ce (propofol Ce from 0 to $6.0 \mu \mathrm{g} / \mathrm{ml}, \mathrm{r}^{2}=-0.83$; propofol Ce from 6.0 to $2.0 \mathrm{\mu g} / \mathrm{ml}, \mathrm{r}^{2}=-0.46$ ). In the spectral analysis of EEG acquired from the PLE monitor, the persistence spectrogram revealed a wide distribution of power at loss of consciousness (LOC) and recovery of consciousness (ROC), with a narrow distribution during unconsciousness. The power spectrogram showed the typical pattern seen in propofol anesthesia with slow alpha frequency band oscillation. The PLE value demonstrated a strong correlation with the BIS value during the change in propofol Ce from 0 to $6.0 \mu \mathrm{g} / \mathrm{ml}\left(r^{2}=0.84\right)$. PLE and BIS values were similar at LOC (62.3 vs. 61.8) $(P>0.05)$, but PLE values were smaller than BIS values at ROC (64.4 vs 75.7) $(P<0.05)$.

Conclusions: The PLE value is a useful anesthetic depth indicator, similar to the BIS value, during propofol anesthesia. Spectral analysis of EEG acquired from the PLE monitor demonstrated the typical patterns seen in propofol anesthesia.

Trial registration: This clinical trial was retrospectively registered at ClinicalTrials.gov at October 2017 (NCT03299621).

Keywords: Anesthetic depth monitoring, Bispectral index, Phase lag entropy, Propofol

\section{Background}

Level of consciousness is related to the complexity and variability of communication between the brain regions [1]. The diverse functional connectivity of the brain in awake state is diminished during anesthesia [2]. There are increases in the phase synchronization or shifts

\footnotetext{
* Correspondence: drhwshin@naver.com

${ }^{1}$ Department of Anesthesiology and Pain Medicine, Korea University Anam Hospital, College of Medicine, Korea University, Goryodae-ro 73, Seongbuk-gu, 02841 Seoul, Republic of Korea

Full list of author information is available at the end of the article
}

between electroencephalography (EEG) signals of the frontal brain during anesthesia, indicating a reduction in communication diversity [3]. The state of consciousness is more closely related to the temporal dynamics of the functional network configuration than to the strength of static connectivity $[4,5]$. The processed EEG signal is an integral part of the brain function monitors used to measure the level of consciousness during anesthesia [6].

Unconsciousness is a fundamental component of general anesthesia; however, anesthesiologists have no reliable way of confirming that a patient is unconscious.

(c) The Author(s). 2020 Open Access This article is distributed under the terms of the Creative Commons Attribution 4.0 International License (http://creativecommons.org/licenses/by/4.0/) which permits unrestricted use, distribution, and reproduction in any medium, provided you give appropriate credit to the original author(s) and the source, provide a link to the Creative Commons license, and indicate if changes were made. The Creative Commons Public Domain Dedication waiver (http://creativecommons.org/publicdomain/zero/1.0/) applies to the data made available in this article, unless otherwise stated. 
Generally, loss of consciousness (LOC) is marked by an increase in low-frequency $(<1 \mathrm{~Hz})$ EEG power, the loss of spatially coherent occipital alpha $(8-12 \mathrm{~Hz})$ oscillations, and the appearance of spatially coherent frontal alpha oscillations [7]; these dynamics are then reversed during recovery of consciousness (ROC) [8]. There have been reports of changes in functional connectivity and disruptions of frontal EEG communication in the brain during anesthesia with propofol $[4,9,10]$, sevoflurane [10-12], and ketamine [10].

The Bispectral Index ${ }^{m a}\left(\right.$ BIS $^{m x}$, Aspect Medical Systems, USA), the most widely used monitor in clinical practice, is based on spectral analysis of frequency powers from one-channel EEG $[4,13,14]$. BIS ${ }^{\mathrm{Tx}}$ is useful for titration of anesthetics and postoperative recovery $[15,16]$. However, BIS monitors cannot provide information regarding functional connectivity in the brain. Previous studies have reported a poor correlation between BIS and depth of anesthesia or sedation $[6,13,17]$.

Phase lag entropy (PLE) is an EEG-based anesthetic depth indicator that calculates diversity in temporal patterns of the phase relationship in the brain $[4,10]$. The recently developed PLE monitor (PLEM ${ }^{\mathrm{mm}}$, Inbody Co., Ltd., Republic of Korea), which measures the PLE value, is a four-channel EEG anesthetic depth monitoring device $[4,18-20]$.

The purpose of this study was to evaluate the clinical performance of the PLEM ${ }^{\mathrm{Tu}}$ to monitor anesthetic depth and to evaluate the correlation between PLE and BIS values during propofol anesthesia.

\section{Methods}

The study was approved by an institutional review board (Korea University Anam Hospital, Institutional Review Board) (IRB No. 2017AN0268), and was prospectively registered, prior to patient enrollment, at ClinicalTrials.gov (NCT03299621, date of registration: October 2017). We also obtained written informed consent from all patients participating in the trial. This study used a prospective, observational, one-group design. The primary end-point of this study was to evaluate the clinical performance of the PLEM ${ }^{\mathrm{ma}}$ to monitor anesthetic depth during propofol anesthesia. The secondary end-point was to evaluate the correlation between the PLE and BIS values during propofol anesthesia.

\section{Anesthesia and monitoring}

Thirty-five adult patients undergoing elective surgery under general anesthesia were enrolled for the study. Patients were aged 20-60 years with an American Society of Anesthesiologists (ASA) physical status I or II. Exclusion criteria were presence of cardiovascular disorders, cerebrovascular disorders, respiratory disorder, and an anticipated difficult airway. Table 1 summarizes the
Table 1 Characteristics of included patients

\begin{tabular}{ll}
\hline Patient characteristics $(n=35)$ & \\
\hline Age (years) & $38.3 \pm 12.2$ \\
Male/Female (number) & $21 / 14$ \\
Weight (kg) & $69.9 \pm 12.5$ \\
Height (cm) & $168.7 \pm 9.6$ \\
ASA I/ASA II (number) & $23 / 12$ \\
Duration of anesthesia (min) & $66.1 \pm 47.5$ \\
Type of surgery & \\
$\quad$ Orthopedic surgery & 24 \\
$\quad$ Genitourinary surgery & 5 \\
Breast surgery & 2 \\
Gynecologic surgery & 4 \\
\hline
\end{tabular}

Data are mean \pm standard deviation or number of patients. American Society of Anesthesiologists (ASA) physical status I; A normal healthy patient, ASA physical status II; A patient with mild systemic disease

patient characteristics. All patients were premedicated with glycopyrrolate $0.2 \mathrm{mg}$ intramuscularly $1 \mathrm{~h}$ before induction of anesthesia. In the operating room, routine standard monitoring was followed, including electrocardiogram, non-invasive blood pressure monitoring, pulse oximetry, capnography, and temperature monitoring. General anesthesia was induced using a propofol targetcontrolled infusion (TCI, Orchestra ${ }^{\circ}$, Fresenius Kabi, France), and intravenous (iv) rocuronium $0.9 \mathrm{mg} / \mathrm{kg}$ was administered for tracheal intubation. To maintain propofol TCI, we used a "staircase" TCI that automatically reached the target effect-site concentrations (Ce) after the propofol Ce was set. After endotracheal intubation, we maintained controlled ventilation with a tidal volume of $6-10 \mathrm{ml} / \mathrm{kg}$, respiration rate of $10-12 / \mathrm{min}$, and inspired oxygen concentration of 0.5. Anesthesia was maintained with remifentanil $\mathrm{Ce} 0-10 \mathrm{ng} / \mathrm{ml}$ (Minto model) and propofol Ce $0-6 \mu \mathrm{g} / \mathrm{ml}$ (Schneider model). Hemodynamics were maintained within a $20 \%$ range of baseline value using fluids, phenylephrine $100-200 \mu \mathrm{g}$ iv $(<20 \%$ from baseline blood pressure), or hydralazine 5$10 \mathrm{mg}$ iv (> 20\% from baseline blood pressure).

\section{Preparation of the PLEM ${ }^{\mathrm{TM}}$ and $\mathrm{BIS}^{\mathrm{TM}}$ sensor}

The PLEM ${ }^{\mathrm{Tm}}$ and BIS ${ }^{\mathrm{Tm}}$ were placed on the left temporalfrontal area, with the BIS ${ }^{\mathrm{sm}}$ sensor above the $\mathrm{PLEM}^{\mathrm{sm}}$ sensor as recommended by the manufacturer (Fig. 1). Both monitors displayed PLE and BIS values and trends, as well as electromyography (EMG) recordings of the forehead muscle, signal quality index (SQI), and real-time EEG waveforms. The anesthesiologist maintained an SQI $>70$ for both devices to ensure accuracy of PLE and BIS values. Both PLEM $^{\mathrm{Tm}}$ and BIS ${ }^{\mathrm{Tm}}$ monitors provide a calculated numeric PLE or BIS value between 0 (isoelectric EEG) and 100 (patient fully awake). The smoothing rates 
of the PLEM ${ }^{\mathrm{TM}}$ and was BIS $^{\mathrm{TM}}$ were $4 \mathrm{~s}$ and $10 \mathrm{~s}$, respectively.

\section{Data collection and EEG acquisition}

Frontal raw EEG signals were recorded using the PLEM ${ }^{\text {тм }}$ sensor. EEG data were recorded with a preamplifier bandwidth of $0.5-45 \mathrm{~Hz}$ and sampling rate of $128 \mathrm{~Hz}$. In order to minimize noise in the EEG signal, we analyzed the data after filtering it through baseline correction and reducing non-specific artifacts. The PLEM ${ }^{\mathrm{TM}}$ electrode array was configured with electrodes positioned approximately at FP1 $\left(\mathrm{L}_{1}\right)$, FP2 $\left(\mathrm{R}_{1}\right)$, AF5 $\left(\mathrm{L}_{2}\right)$, and AF6 $\left(\mathrm{R}_{2}\right)$. The ground electrode was at Fpz, and the reference electrode was at position $\mathrm{T} 3$ on the temporal area of the face (T) (Fig. 1). Electrode impedance was less than $7 \mathrm{k} \Omega$ in each channel.

\section{Data measurement at time points}

We collected data regarding patient demographics and anesthetic management. We acquired the data for PLE value, EEG band power, and BIS value using a USB memory card on PLEM ${ }^{\mathrm{TM}}$ and $\mathrm{BIS}^{\mathrm{TM}}$ devices at the following points in time: (1) prior to intubation as propofol Ce increased from $0 \mu \mathrm{g} / \mathrm{ml}$ to $2,3,4,5$, and $6 \mu \mathrm{g} / \mathrm{ml}$, and after intubation as propofol Ce decreased from $6 \mu \mathrm{g} / \mathrm{ml}$ to $5,4,3$, and $2 \mu \mathrm{g} / \mathrm{ml}$; (2) two min after muscle relaxant injection and tracheal intubation; (3) at LOC, defined as when a patient no longer responds to the verbal command "open your eyes" (modified Observer's Assessment Alertness/Sedation $[\mathrm{OAA} / \mathrm{S}]$ scale $=2$ ) repeated every 10 s during induction; and (4) at ROC, defined as when a patient once again obeys the verbal command "open your eyes" (modified OAA/S scale $=3$ ) repeated every $10 \mathrm{~s}$ during emergence from anesthesia.

\section{Calculation of PLE}

The PLE value was calculated following the approach used in Lee et al.'s study [4]. The degree of communication between different areas of the brain is correlated with the phase relationship among multi-channel EEG signals $[8,15]$. In order to calculate the PLE value, the data from the frontal and prefrontal lobes were recorded using PLEM $^{\mathrm{TM}}$ sensor electrodes arranged at FP1, FP2, AF5, and AF6 (Fig. 1). EEG signals were segmented into 4-s time series with 50\% overlapping epochs. All filters used a zero-phase finite impulse response to prevent changes in phase. In addition, the correction algorithm employing nonlinear signal decomposition was used to correct the amplitude and base line of the signals. Calculations were performed after removal of signals outside the range of biological noise and EEG signals. Because the amplitude of noise is also physiologically significant, we used correction methods to limit the elimination of noise. However, the calculation was not performed if the

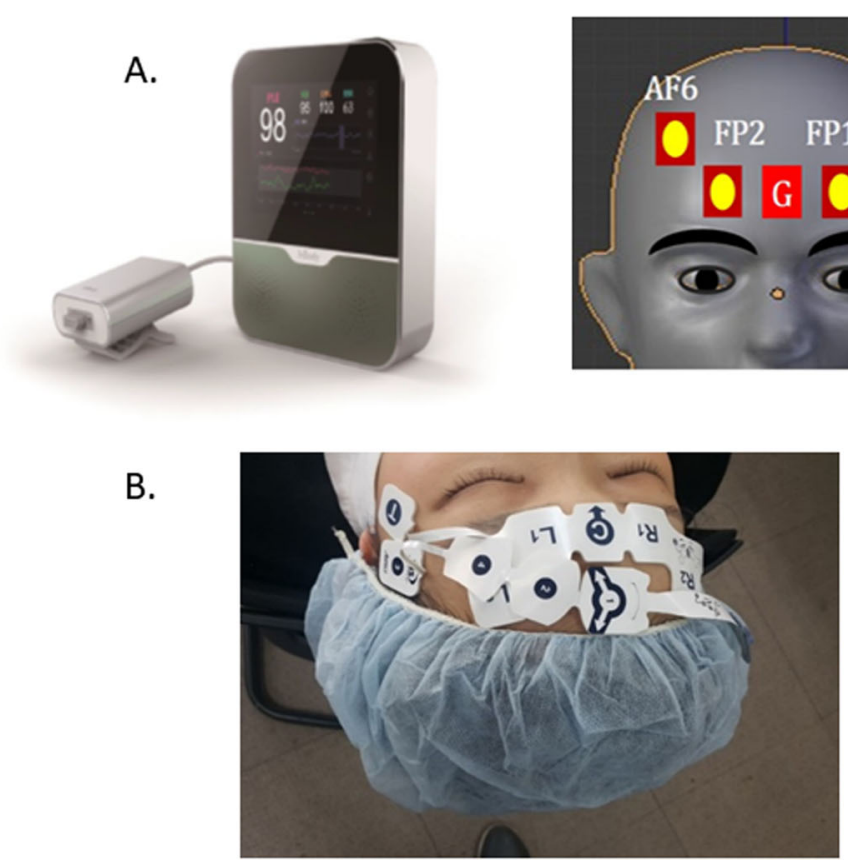

Fig. 1 Illustration of electroencephalogram (EEG) channels and phase difference sign measurement. a PLEM ${ }^{\mathrm{TM}}$ measures the phase lag entropy (PLE) value. The PLE electrode array consists of electrodes positioned approximately at FP1 $\left(L_{1}\right)$, FP2 $\left(R_{1}\right)$, AF5 $\left(L_{2}\right)$, and AF6 $\left(R_{2}\right)$. The ground electrode is at $\mathrm{F}_{\mathrm{PZ}}(\mathrm{G})$, and the reference electrode is at $\mathrm{T3}$ of the temporal area of the face $(\mathrm{T})$. $\mathbf{b}$ Both sensors are placed on the left temporalfrontal area with the bispectral index (BIS) sensor placed above the PLE sensor. Permitted for the copyright for PLEM ${ }^{\mathrm{TM}}$ monitor image [15, 01, 2020] by Copyright Holder (InBody co., Itd) 
data exceeded $50 \%$ of the epoch. PLEM ${ }^{\mathrm{m}}$ operates by extracting and combining EEG signals from the frontal and prefrontal regions. The instantaneous phase was extracted via Hilbert transform using the signal processing toolbox in MATLAB (version 2017b, Mathworks Inc., Co., Ltd., USA). The PLE value was quantified using the entropy of regularity or irregularity in the temporal variation of phase difference between two EEG signals. In order to calculate the PLE value, the instantaneous phase signal was extracted from two signals and the difference value of the instantaneous phase was encoded. $S_{t}=1$ if $\Delta \phi_{t}>0$ (i.e., first signal is phase leading the second signal), and $S_{t}=0$ if $\Delta \phi_{t}<0$ (i.e., first signal is phase lagging the second signal). Thus, the vector $S_{t}$, representing the temporal pattern of the phase relationship is given by

$$
\begin{aligned}
S_{t} & =\left\{s_{t}, s_{t+\tau}, \ldots s_{t+(m-1) \tau}\right\} t \\
& =1,2, \ldots, N-(m-1) \tau
\end{aligned}
$$

where, $m$ and $s$ represent pattern size (word length) and time lag, respectively. For example, with $m=3$, eight patterns (“000," "001," “010," “100," "011," "101," “110," and "111") can be generated. Finally, the PLE value was calculated by applying the standard Shannon entropy formula for the distribution of the phase patterns:

$$
\mathrm{PLE}=-\frac{\sum p_{j} \log p_{j}}{\log \left(2^{m}\right)}
$$

In Eq. (2), $\mathrm{p}_{i}$ represents the probability of the occurrence of the $j^{\text {th }}$ pattern in a given input signal, and $\mathrm{m}$ represents the size of one pattern. Eq. (2) is in the form of a fraction, where the numerator is the entropy of the probability of different phase patterns occurring in the signal, and the denominator is the number of all possible patterns. The normalization term in the denominator scale of the PLE value is the range [0 1]. PLEM ${ }^{\mathrm{Tm}}$ displays the index value on the screen in a linear scale $(\times 100)$ with a value between 0 and 100. The PLE is an algorithm designed to reflect the functional connectivity of the frontal area in the brain. In the awake state, the histogram distribution of patterns is relatively even and thus has a high PLE value. In the sedated state, the distribution of patterns is biased toward a low PLE value.

\section{Spectral analysis}

We used spectral analysis to analyze whether the EEG signal acquired from PLEM ${ }^{\mathrm{su}}$ was consistent with the typical known patterns in the persistence and power spectrograms during propofol anesthesia $[7,12]$.

The persistence spectrogram was analyzed using MATLAB. We divided the EEG signal into segments with a uniform epoch length (4s), then overlapped the spectrogram $(2 \mathrm{~s})$ such that the frequency power at each frequency $(0.125 \mathrm{~Hz})$ represented a high percentage of the spectrogram. EEG signals were divided into three states during propofol anesthesia: awake state (A-state), unconscious state (UC-state), and recovery of consciousness state (ROC-state). The frequency and frequency power were plotted on the $\mathrm{x}$-axis and $\mathrm{y}$-axis, respectively. The distribution of power was shown using color to visually represent a decibel ratio (\%) [21].

For the power spectrogram, we estimated the standard Multitaper Power Spectral Density (MPSD) using MATLAB [22]. For computing the power spectrogram, we obtained individual four-channel EEG signals acquired from PLEM ${ }^{\mathrm{Tx}}$. We computed four-channel median spectrograms by taking the median across all time epochs. The time was plotted on the $\mathrm{x}$-axis and frequency on the $y$-axis; the signal frequency power was expressed in scale color. The power spectrogram quantifies the frequency distribution of energy or power within the EEG signal over time. We calculated the MPSD using 8-s EEG segments ( $4 \mathrm{~s}$ before to $4 \mathrm{~s}$ after each EEG measurement point) to quantify the frequency power ratio for a given propofol Ce. We set the following parameters: window length (2 s), overlap (1 s), time-half bandwidth product $(3 \mathrm{~Hz})$, and spectral resolution $(0.25$ $\mathrm{Hz})$. We calculated the average of the listed fourchannel MPSD values for all band power values.

\section{Calculation of EEG band power}

We also computed the ratio of EEG band powers (gamma, beta, alpha, theta, and delta) using the following equation for each time point with propofol $\mathrm{Ce}$ in all patients:

$$
\begin{aligned}
& \text { Ratio of EEG band power }(\gamma, \beta, \alpha, \theta, \delta) \\
& =\frac{\text { Frequency band power }(\gamma, \beta, \alpha, \theta, \delta)}{\text { Total frequency power }}
\end{aligned}
$$

The absolute values of EEG-derived band power in each patient were calculated using the ratio to reduce the effect of differences between patients.

\section{Statistical analysis}

Statistical analysis was performed using SPSS software version 20 (IBM Corporation, Armonk, NY, USA). A correlation coefficient of 0.7 , for the index of the anesthetic depth monitoring device according to the propofol Ce, was considered to be clinically significant. We calculated a minimum requirement of 29 patients. We also calculated a sample size of 32 based on previous observational studies correlating EEG-based anesthetic depth monitoring (a difference of 10 between BIS and entropy based on pilot study, a power of $90 \%$ with an $\alpha$ value of 0.05) [23]. Considering possible data loss, we decided to study 35 patients. 
The data are presented as mean $\pm \mathrm{SD}$ or median for continuous variables, and number of patients for categorical variables. Spearman correlation analysis was performed between the PLE value and propofol $\mathrm{Ce}$ and displayed using box and whiskers plots. Repeated measures analysis of variance (ANOVA) was performed for changes in PLE value before and after muscle relaxant injection, and for the change in EEG-band power during propofol anesthesia, displayed using box and whiskers plots. Post-hoc multiple comparisons were then performed using the Tukey test. A comparison between the PLE value and BIS value at the same points in time was performed using the $t$-test with the Bland-Altman graph. $P$-values $<0.05$ were considered statistically significant.

\section{Results}

Of the 35 patients, one was excluded because of a technical error during recording with the sensor electrode. Therefore, 34 patients were included in the statistical analysis. The demographic data of these patients are shown in Table 1.

\section{Correlation between PLE value and changes in propofol}

$\mathrm{Ce}$

The PLE values were inversely correlated to changes in propofol Ce (propofol Ce from 0 to $6.0 \mu \mathrm{g} / \mathrm{ml}$, Spearman correlation coefficient $\mathrm{r}^{2}=-0.835$; propofol Ce from 6.0 to $2.0 \mu \mathrm{g} / \mathrm{ml}, \mathrm{r}^{2}=-0.467$ ) (Fig. 2a). The PLE value at LOC was $62.3 \pm 10.9$ with propofol Ce $4.4 \pm 0.8 \mu \mathrm{g} / \mathrm{ml}$, whereas the PLE value at ROC was $64.4 \pm 9.6$ with propofol Ce $1.0 \pm 0.2 \mu \mathrm{g} / \mathrm{ml}$.

\section{Spectral analysis of EEG acquired from PLEM ${ }^{\mathrm{Tm}}$}

The persistence spectrogram showed a wide distribution of power for a given frequency in the LOC-state and ROC-state compared to the dense distribution of power for a given frequency in the UC-state (Fig. 3a). The power spectrogram showed a typical pattern of propofol-induced sedation with slow alpha $(8-12 \mathrm{~Hz})$ band oscillations (Fig. 3b) [7]. PLE and BIS values were inversely proportional to propofol Ce (Fig. 3c). For each EEG band power, as propofol Ce increased, the alpha-, beta-, and theta-band powers showed a tendency to increase, and the gamma-band power showed a tendency to decrease. There was no significant change in the delta-band power. However, the changes in EEG band power were not statistically significant because of the wide range of SD and some outliers (Fig. 4).

\section{A comparison of PLE and BIS}

The PLE value demonstrated a strong correlation with the BIS value during the change in propofol Ce from 0 to $6.0 \mu \mathrm{g} / \mathrm{ml} \quad\left(\mathrm{r}^{2}=0.84\right)$ (Fig. 5). The PLE was significantly higher than the BIS at all propofol Ce values prior to intubation $(P<0.05)$, and lower than the BIS at all propofol Ce values after intubation $(\mathrm{P}<0.05)$ (Table 2$)$ (Fig. 5). The PLE values were similar to the BIS at LOC (PLE: $62.3 \pm 10.9$, BIS: $61.8 \pm 10.5$ ), but lower at ROC (PLE: 64.4 \pm 9.6, BIS: $75.7 \pm 6.4)(\mathrm{P}<0.05)$.

\section{Effect of muscle relaxants}

Both PLE and BIS values significantly decreased after injection of the muscle relaxant (PLE: $43.8 \pm 11.6$ vs $38.3 \pm$ 4.2, BIS: $49.7 \pm 14.5$ vs $36.5 \pm 9.7)(\mathrm{P}<0.05)$.
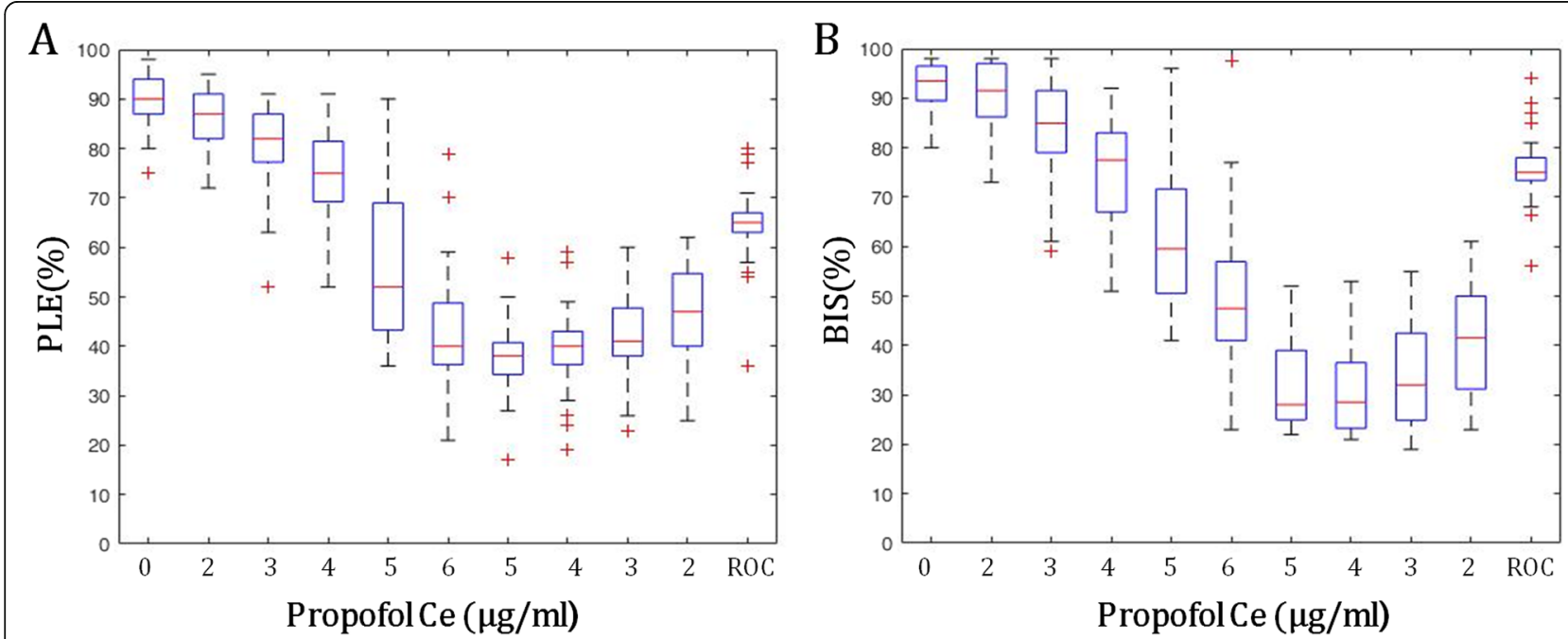

Fig. 2 The change in PLE and BIS values during propofol anesthesia. The box and whiskers plots show PLE and BIS values at the time when a given propofol target effect concentration (propofol Ce) was reached. a For PLE, Spearman correlation coefficient $=0.835$ (from propofol Ce 0 to propofol Ce $6.0 \mu \mathrm{g} / \mathrm{ml}$ ). b For BIS, Spearman correlation coefficient = 0.781 (from propofol Ce 0 to propofol Ce $6.0 \mu \mathrm{g} / \mathrm{ml}$ ). Abbreviations: PLE, phase lag entropy; BIS, bispectral index; LOC, loss of consciousness; ROC, recovery of consciousness. The boxes depict the median values and the 25th and the 75th percentiles (lower whisker $=-1.5 \times 1 \mathrm{IQR}$, upper whisker $=+1.5 \times 1 \mathrm{QR}$, IQR; inter-quartile range, + ; outlier) 


\section{[Group persistence spectrum]}
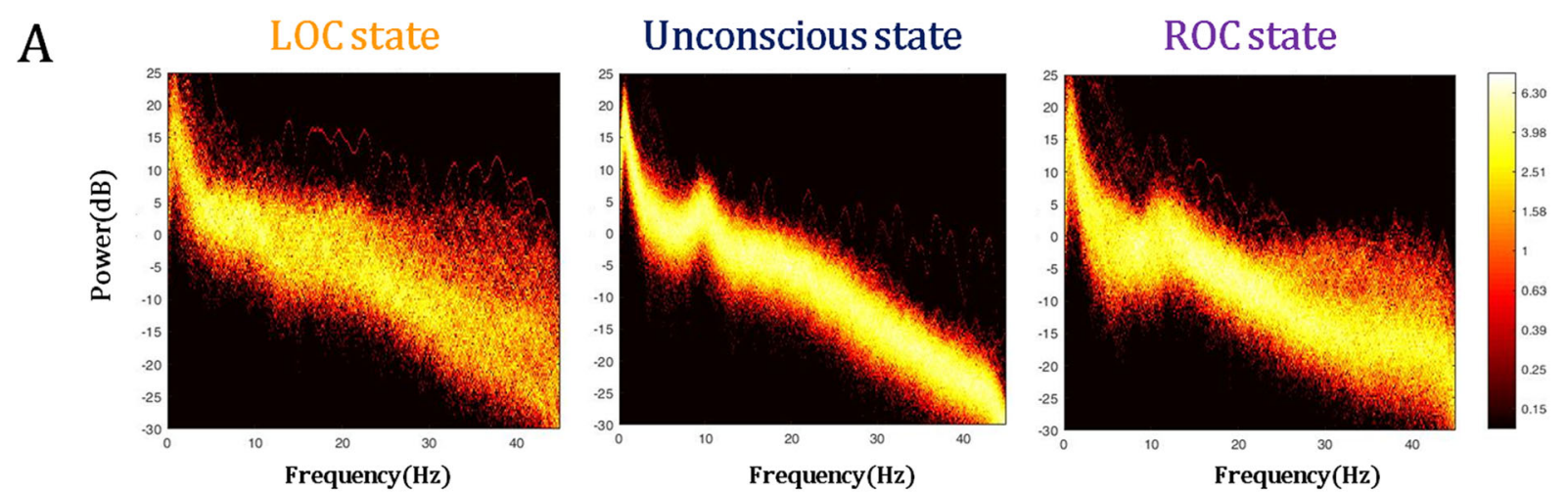

B

[Represntative Spectrogramand Phase lag entropy]

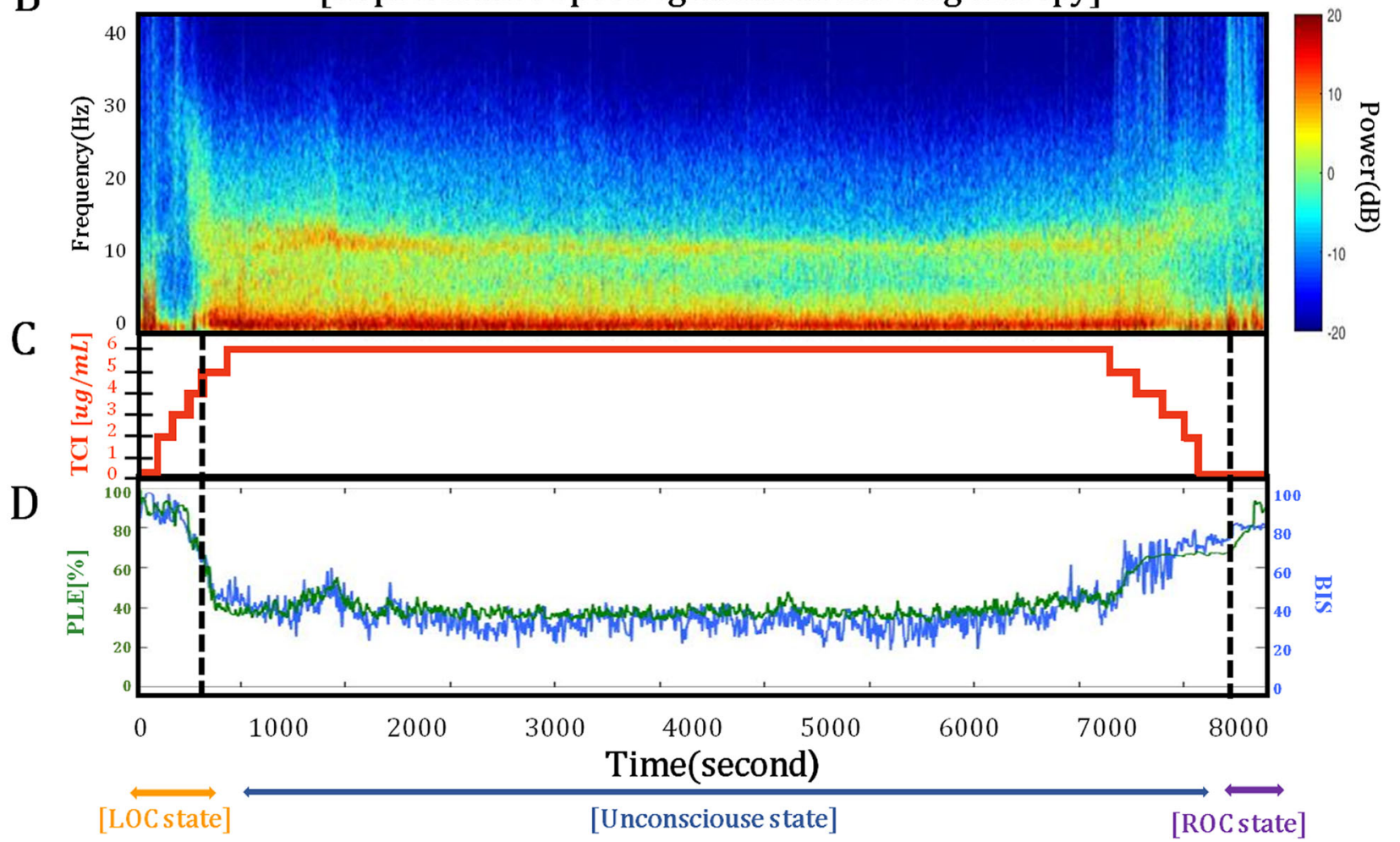

Fig. 3 The spectrograms obtained from PLEM ${ }^{\top M}$ during propofol anesthesia. a Group-level persistence spectrogram for different states of consciousness (LOC-state, UC-state, and ROC-state). b Representative power spectrogram showing the power of the slow and delta (0.1 to $4 \mathrm{~Hz}$ ) and alpha $\left(8\right.$ to $13 \mathrm{~Hz}$ ) band oscillations, and $\mathbf{c}$ the time domain phase lag entropy (PLE) obtained from PLEM ${ }^{\mathrm{TM}}$ during propofol anesthesia. Abbreviations: TCl, target-controlled infusion; LOC, loss of consciousness; UC, unconsciousness; ROC, recovery of consciousness. The black dashed line indicates when LOC and ROC occurred

\section{Discussion}

In this study, PLE values from PLEM ${ }^{\mathrm{Tm}}$ were inversely correlated to changes in propofol Ce during the induction and emergence period of propofol anesthesia. The persistence spectrogram and power spectrogram using EEG signals acquired from the PLEM ${ }^{\mathrm{rm}}$ were consistent with the typical known patterns seen in propofol anesthesia. The PLEM $^{\mathrm{TM}}$ was similarly comparable for anesthetic depth monitoring with $\mathrm{BIS}^{\mathrm{mi}}$ during the change of propofol Ce 0 to Ce $6.0 \mu \mathrm{g} / \mathrm{ml}$. The PLE value was lower at BIS values above 40, but higher at BIS values below 40. The PLE and BIS values were similar at LOC, but the PLE values were smaller than the BIS values at ROC.

To date, there have been many studies for developing a method of assessing anesthetic depth using processed EEG data [8]. The most widely used processed EEGbased anesthetic depth monitor, BIS ${ }^{\mathrm{Tw}}$, quantifies the consistency of phase-coupling and frequency of single- 


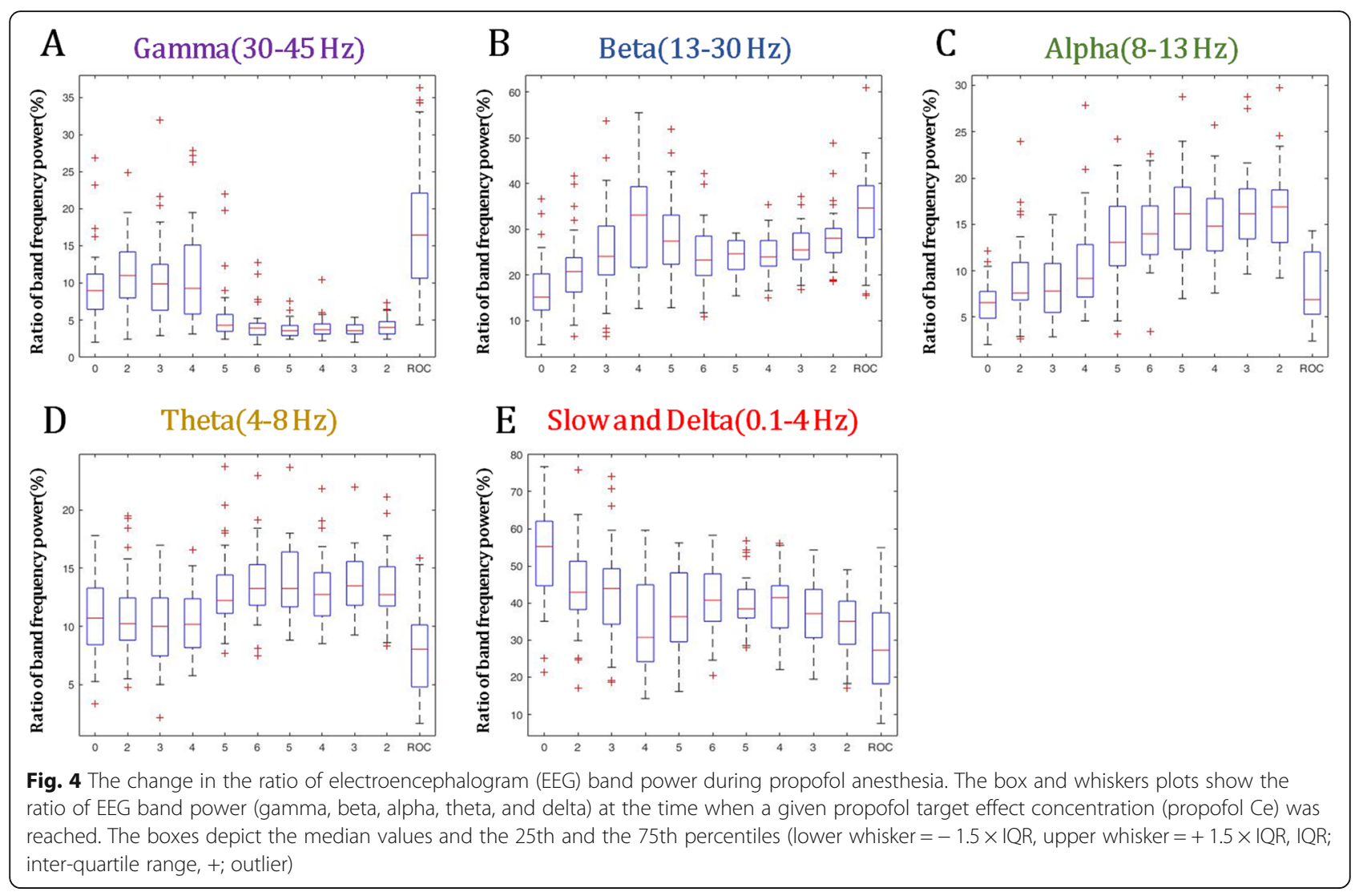

channel EEG in the brain, whereas PLEM ${ }^{\mathrm{Tm}}$ quantifies the entropy based on the spatial or connectivity information of four-channel EEG signals by measuring the regularity of variations in the temporal phase difference between two separate areas of the brain $[4,8]$.

PLEM $^{\mathrm{m}}$ is a recently developed anesthetic depth monitoring device that uses four-channel EEG [4]. The PLE value in PLEM $^{\mathrm{Tm}}$ is composed of three sub-parameters, PLE1 $(8-32 \mathrm{~Hz})$, PLE2 $(0.1-1$ and $32-45 \mathrm{~Hz})$, and BSR
(2-32 Hz). PLE1 (light hypnotic state) is calculated from the alpha $(8-13 \mathrm{~Hz})$ and beta $(13-30 \mathrm{~Hz})$ bands, whereas PLE2 (deep hypnotic state) is calculated from the slowfrequency $(0.1-1 \mathrm{~Hz})$ and gamma $(30-45 \mathrm{~Hz})$ bands. BSR is composed of two types of burst-suppression detection, such as the portions of the isoelectric EEG and/ or a very low power frequency. The PLE value (scale 0 100 ) is calculated by combining PLE1, PLE2, and BSR with appropriate weights.
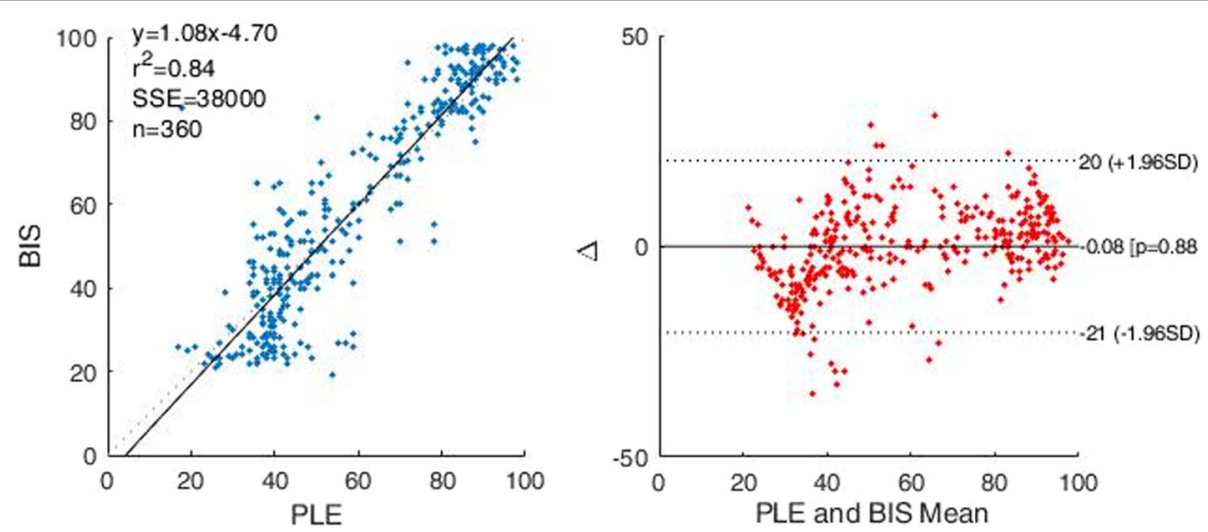

Fig. 5 A comparison of PLE and BIS using the Spearman correlation graph and Bland-Altman graph. Abbreviations: PLE, phase lag entropy; BIS, bispectral index; SD, standard deviation 
Table 2 PLE and BIS values at the time point when propofol reached the target effect concentration

\begin{tabular}{llll}
\hline Propofol Ce $(\mu \mathrm{g} / \mathrm{ml})$ & PLE & BIS & P-value \\
\hline 0 & $89.1 \pm 8.5^{*}$ & $91.6 \pm 5.7$ & 0.042 \\
2 & $83.9 \pm 12.7^{*}$ & $90.7 \pm 6.4$ & 0.002 \\
3 & $79.0 \pm 10.7^{*}$ & $83.5 \pm 10.7$ & 0.000 \\
4 & $74.5 \pm 9.2$ & $74.9 \pm 11.1$ & 0.798 \\
5 & $56.8 \pm 14.8^{*}$ & $62.0 \pm 14.8$ & 0.001 \\
6 & $43.7 \pm 11.4^{*}$ & $49.7 \pm 14.5$ & 0.002 \\
Intubation & & & \\
5 & $37.3 \pm 7.1^{*}$ & $31.6 \pm 8.2$ & 0.005 \\
4 & $39.3 \pm 7.8^{*}$ & $30.8 \pm 8.8$ & 0.000 \\
3 & $41.7 \pm 9.1^{*}$ & $34.5 \pm 10.7$ & 0.000 \\
2 & $47.2 \pm 9.1^{*}$ & $41.1 \pm 11.6$ & 0.000
\end{tabular}

Data are mean \pm standard deviation or values. Abbreviations: PLE, phase lag entropy; BIS, bispectral index; propofol $\mathrm{Ce}$, propofol target effect-site concentration. * $P$-value $<0.05$ compared with BIS at the same time points for propofol Ce 0-6 $\mu \mathrm{g} / \mathrm{ml}$

In this study, we found that the PLE values were inversely correlated to the changes in propofol Ce when propofol Ce was increased $\left(\mathrm{r}^{2}=-0.835\right)$ and decreased $\left(r^{2}=-0.467\right)$. The PLE values at LOC and ROC were similar (62.3 and 64.4, respectively). Koo et al.'s study [24] results of propofol Ce at LOC and ROC (4.4 \pm $1.1 \mu \mathrm{g} / \mathrm{ml}, 1.1 \pm 0.3 \mu \mathrm{g} / \mathrm{ml}$ ) are similar to that in our study. In Lee et al.'s study [4] that compared recently used anesthetic depth monitors, the PLE value exhibited the highest agreement with the level of consciousness (using the modified OAA/S score) relative to other monitors such as the BIS, relative beta ratio (RBR), approximate entropy (ApEn), and permutation entropy (PeEn) values. Recently, clinical studies have been reported for PLEM $^{\mathrm{Ta}}$ during propofol-induced sedation [18-20]. Jung et al. [20] reported that PLEM ${ }^{\mathrm{Tm}}$ was comparable with BIS $^{\text {Tu }}$ in correlational studies using the OAA/S score during propofol-induced sedation (Spearman's Rho: 0.755 for PLE, 0.788 for BIS). Ki et al. [18] also reported the pharmacodynamic modeling for each OAA/S score using PLE values from the PLEM ${ }^{\mathrm{mm}}\left(\mathrm{Ce}_{50}\right.$ value: $1.67 \mu \mathrm{g} /$ $\mathrm{ml}, 1.96 \mu \mathrm{g} / \mathrm{ml}, 2.22 \mu \mathrm{g} / \mathrm{ml}$, and $2.69 \mu \mathrm{g} / \mathrm{ml}$ for OAA $/ \mathrm{S}$ scores of $\leq 4, \leq 3, \leq 2$, and $\leq 1$, respectively). Therefore, based on the above study results, the PLEM ${ }^{\mathrm{m}}$ may be used for monitoring anesthetic depth during propofol anesthesia.

Propofol reduces the excitation potential input to the cortex by binding to post-synaptic $\gamma$-aminobutyric acid A $\left(\mathrm{GABA}_{\mathrm{A}}\right)$ receptors $[8,25]$. Modeling and experimental studies using propofol suggest that the potentiation of GABA receptors leads to a state of thalamo-cortical synchrony associated with unconsciousness, observed as frontal slowing and alpha band oscillations [8]. In the states bordering consciousness, such as LOC, the power of alpha and beta bands in EEG waves move from the occipital region to the frontal region. This is a recognized phenomenon called "anteriorization" $[7,8,15,26]$. Coherent alpha oscillations and disruption of neural spiking activity associated with slow oscillations are the two main mechanisms of propofol-induced sedation [7, 27, 28].

We used spectral analysis of the persistence spectrogram and power spectrogram to see whether the EEG signal acquired from PLEM $^{\mathrm{Tm}}$ reflected typical known patterns of propofol anesthesia that had been reported in previous studies $[7,12,29]$. The persistence spectrogram showed wide distribution in the LOC-state and ROC-state, in contrast to a dense distribution in the UC-state. The power spectrogram also showed a typical pattern with slow alpha $(8-12 \mathrm{~Hz})$ band oscillations [7].

Using PLEM ${ }^{\mathrm{m}}$, we could measure EEG band power (the ratio of gamma, beta, alpha, theta, and delta waves). In previous studies of EEG band power during propofolinduced sedation, it was found that as the modified $\mathrm{OAA} / \mathrm{S}$ score decreases, the power of the alpha-beta band gradually increases, and the delta-band power increases after LOC $[7,12,30]$. We also observed the same results in our study; as propofol Ce increased, alpha-, beta-, and theta- band powers showed the tendency to increase, and gamma-band power showed the tendency to decrease. However, these changes were not statistically significant because of the wide SD and some outliers. Generally, as the anesthetic depth increases, the frequency of the EEG band power shifts toward the lower frequencies (beta to alpha to theta to delta). If the propofol Ce was increased any further in our study, burst suppression activity would ensue [7]. In our study, the gamma-band power increased during the start of propofol TCI and then decreased at propofol Ce 4-5 $\mu \mathrm{g}$ $/ \mathrm{ml}$; this might be because of noise interference with the EEG [25].

Before the start of our study, by prioritizing spatial concepts using four-channel EEG in PLEM ${ }^{\mathrm{Tm}}$, we hypothesized that the PLE value might reflect the anesthetic depth better than the other widely used anesthetic depth monitors, such as the BIS value. In our study, the PLE value demonstrated a strong correlation with the BIS value. The PLE was significantly higher than the BIS prior to intubation, and lower than the BIS after intubation. The PLE values were similar to the BIS at LOC (PLE: 62.3, BIS: 61.8), but lower to the BIS at ROC (PLE: 64.4, BIS: 75.7). In the previous studies [31-33], the BIS values were affected by the degree of neuromuscular block. Contrary to the BIS, the PLE is less influenced by EMG signal. In PLE algorithm, noise introduced into both channel $(R 1, R 2)$ at the same time is removed during the binarization process ("000," "001," "010," "100," "011," "101," "110," and "111") of phase difference by 
phase extraction. The EMG activity in our result was higher in ROC compared to LOC $(27.9 \pm 41.7 \%$ vs. $67.4 \pm 22.5 \%$ ). We suspect the observed differences between the BIS and PLE, especially during ROC phase, may be due to the difference in the EMG-EEG interferences of both devices. However, in the results, both PLE and BIS values significantly decreased after injection of the muscle relaxant. Further studies are suggested for the effect of EMG signals on the PLE and the BIS.

The limitations of our study are as follows. First, BIS sensor (circle 3) was slightly mal-positioned (the commercially recommended location of the BIS sensor is as follows: circle 1 at the center of the forehead, approximately $4 \mathrm{~cm}$ above the nose, circle 2 at $2.8 \mathrm{~cm}$ lateral right to circle 1 , and circle 3 on the temple area between the corner of the eye and the hairline). Second, the disagreement between the PLE value and the BIS value at each time point showed large standard deviation. Third, the PLE and BIS values were not measured at the same point in time because of the different smoothing rates of the devices. The smoothing rates for PLE and BIS were $4 \mathrm{~s}$ and $10 \mathrm{~s}$, respectively. Fourth, PLE and BIS values changed continuously after reaching propofol Ce. The inter-trial variability of these values was related to time. Fifth, we only investigated the performance of PLEM ${ }^{\mathrm{Tm}}$ in young adults during propofol anesthesia [7]. Further studies are needed to validate the PLE value from $\mathrm{PLEM}^{\mathrm{sm}}$ for pediatric or geriatric patients and with other drugs such as other GABAnergic anesthetic drugs and non-GABAnergic drugs such as ketamine, dexmedetomidine, and $\mathrm{N}_{2} \mathrm{O}$.

\section{Conclusion}

The PLE value obtained using PLEM ${ }^{\mathrm{Tm}}$ is a useful anesthetic depth indicator, similar to the BIS value, in patients subjected to propofol anesthesia. Spectral analysis of the raw EEG signals acquired from the PLEM ${ }^{\mathrm{m}}$ demonstrated the typical patterns of propofol anesthesia.

\section{Abbreviations}

ANOVA: Analysis of variance; ApEn: Approximate entropy; ASA: American Society of Anesthesiologists; A-state: Awake state; BIS: Bispectral index; Ce: Target effect-site concentration; EEG: Electroencephalography; EMG: Electromyography; GABA: $\gamma$-aminobutyric acid; LOC: Loss of consciousness; MPSD: Multitaper power spectral density; OAA/S: Observer's Assessment Alertness/Sedation; PeEn: Permutation entropy; PLE: Phase lag entropy; RBR: Relative beta ratio; ROC: Recovery of consciousness; ROCstate: Recovery of consciousness state; SQI: Signal quality index; TCl: Targetcontrolled infusion; UC-state: Unconscious state

\section{Acknowledgments}

We thank Kyoung Soo Kim (Neuroscience researcher, InBody, Seoul, Korea) for the support in the data analysis. We would like to thank Editage (www. editage.co.kr) for English language editing.

\section{Authors' contributions}

Study conception and design: HW Shin, HJ Kim. Experiment performed by: HW Shin, HJ Kim, YK Jang, HS You. Data acquisition: HJ Kim, YK Jang, YJ Choi, H Huh, JS Hong. Data analysis: YK Jang, HW Shin, SU Choi. Data processing and data interpretation: HW Shin, SU Choi, H Huh. Statistical analysis: HW Shin, JS Hong, HS You. Manuscript drafted by: HW Shin, YJ Choi, JS Hong. Draft revised critically for important intellectual content by: HW Shin. All authors read and approved the final manuscript.

\section{Funding}

This study was funded from Inbody Co., Ltd. Inbody paid for the research grant to our institution. Inbody Co. supplied the study device (PLE monitor) free of charge. The funding body had the role for the data analysis.

\section{Availability of data and materials}

The dataset generated and analyzed during the current study is available from the corresponding author on reasonable request.

\section{Ethics approval and consent to participate}

This prospective, observational study was approved by the Institutional Review Board of Korea University Anam Hospital (2017AN0268). Written informed consent of patients was obtained according to the Declaration of Helsinki. We also registered in ClinicalTrials.gov (NCT03299621).

\section{Consent for publication}

Not applicable.

\section{Competing interests}

The authors declare that they have no competing interests.

\section{Author details}

${ }^{1}$ Department of Anesthesiology and Pain Medicine, Korea University Anam Hospital, College of Medicine, Korea University, Goryodae-ro 73, Seongbuk-gu, 02841 Seoul, Republic of Korea. ${ }^{2}$ Department of

Anesthesiology and Pain Medicine, Ewha University Magok Hospital, College of Medicine, Ewha University, Seoul, Republic of Korea. ${ }^{3}$ Department of Anesthesiology and Pain Medicine, Korea University Ansan Hospital, College of Medicine, Korea University, Gyeonggi-do, Republic of Korea.

Received: 14 September 2019 Accepted: 19 February 2020

Published online: 26 February 2020

\section{References}

1. Dehaene S, Changeux JP. Experimental and theoretical approaches to conscious processing. Neuron. 2011;70:200-27.

2. Alkire MT, Hudetz AG, Tononi G. Consciousness and anesthesia. Science. 2008:322:876-80.

3. Lee U, Kim S, Noh GJ, Choi BM, Hwang E, Mashour GA. The directionality and functional organization of frontoparietal connectivity during consciousness and anesthesia in humans. Conscious Cogn. 2009;18:1069-78.

4. Lee H, Noh GJ, Joo P, Choi BM, Silverstein BH, Kim M, et al. Diversity of functional connectivity patterns is reduced in propofol-induced unconsciousness. Hum Brain Mapp. 2017;38:4980-95.

5. Barttfeld P, Uhrig L, Sitt JD, Sigman M, Jarraya B, Dehaene S. Signature of consciousness in the dynamics of resting-state brain activity. Proc Natl Acad Sci. 2015;112:887-92

6. Fahy BG, Chau DF. The Technology of Processed Electroencephalogram Monitoring Devices for assessment of depth of anesthesia. Anesth Analg. 2018;126:111-7

7. Purdon PL, Sampson A, Pavone KJ, Brown EN. Clinical electroencephalography for anesthesiologists. Anesthesiology. 2015;123: 937-60

8. Purdon PL, Pierce ET, Mukamel EA, Prerau MJ, Walsh JL, Wong KFK, et al. Electroencephalogram signatures of loss and recovery of consciousness from propofol. Proc Natl Acad Sci. 2013;110:E1142-51.

9. Hudetz AG, Liu X, Pillay S. Dynamic repertoire of intrinsic brain states is reduced in Propofol-induced unconsciousness. Brain Connect. 2015:5:10-22.

10. Lee U, Ku S, Noh G, Baek S, Choi B, Mashour GA. Disruption of frontalparietal communication by ketamine, Propofol, and Sevoflurane. Anesthesiology. 2013;118:1264-75.

11. Ward LM, Ku S-W, Lee U, Noh G-J, Jun I-G, Mashour GA. Preferential inhibition of frontal-to-parietal feedback connectivity is a neurophysiologic correlate of general anesthesia in surgical patients. PLoS One. 2011;6: e25155. 
12. Akeju O, Westover MB, Pavone K, Sampson AL, Hartnack KE, Brown EN, et al. Effects of Sevoflurane and Propofol on frontal electroencephalogram power and coherence. Anesthesiology. 2014;121:990-8.

13. Hajat Z, Ahmad N, Andrzejowski J. The role and limitations of EEG-based depth of anaesthesia monitoring in theatres and intensive care. Anaesthesia. 2017;72:38-47.

14. Johansen JW. Update on bispectral index monitoring. Best Pract Res Clin Anaesthesiol. 2006;20:81-99.

15. Cimenser A, Purdon PL, Pierce ET, Walsh JL, Salazar-Gomez AF, Harrell PG, et al. Tracking brain states under general anesthesia by using global coherence analysis. Proc Natl Acad Sci. 2011;108:8832-7.

16. Punjasawadwong Y, Phongchiewboon A, Bunchungmongkol N. Bispectral index for improving anaesthetic delivery and postoperative recovery. Cochrane Database Syst Rev. 2014;17:CD003843.

17. Nasraway SA, Wu EC, Kelleher RM, Yasuda CM, Donnelly AM. How reliable is the Bispectral index in critically ill patients? A prospective, comparative, single-blinded observer study. Crit Care Med. 2002;30:1483-7.

18. Ki S, Kim KM, Lee YH, Bang JY, Choi BM, Noh GJ. Phase lag entropy as a hypnotic depth indicator during propofol sedation. Anaesthesia. 2019;74: 1033-40.

19. Seo KH, Kim KM, Lee SK, John H, Lee J. Comparative analysis of phase lag entropy and Bispectral index as anesthetic depth indicators in patients undergoing thyroid surgery with nerve integrity monitoring. J Korean Med Sci. 2019;34:e151.

20. Jun MR, Yoo JH, Park SY, Na S, Kwon H, Nho J-H, et al. Assessment of phaselag entropy, a new measure of electroencephalographic signals, for propofol-induced sedation. Korean J Anesthesiol. 2019;72:351.

21. Fulop SA, Fitz K. Algorithms for computing the time-corrected instantaneous frequency (reassigned) spectrogram, with applications. J Acoust Soc Am. 2006;119:360-71.

22. Babadi B, Brown EN. A review of multitaper spectral analysis. IEEE Trans Biomed Eng. 2014;61:1555-64.

23. Musialowicz T, Lahtinen P, Pitkänen O, Kurola J, Parviainen I. Comparison of spectral entropy and BIS VISTA ${ }^{\text {TM }}$ monitor during general anesthesia for cardiac surgery. J Clin Monit Comput. 2011:25:95-103.

24. Koo BN, Lee JR, Noh GJ, Lee JH, Kang YR, Han DW. A pharmacodynamic analysis of factors affecting recovery from anesthesia with propofolremifentanil target controlled infusion. Acta Pharmacol Sin. 2012;33:1080-4.

25. Hemmings HC Jr, Akabas MH, Goldstein PA, Trudell JR, Orser BA, Harrison NL. Emerging molecular mechanisms of general anesthetic action. Trends Pharmacol Sci. 2005;26:503-10.

26. Tinker JH, Sharbrough FW, Michenfelder JD. Anterior shift of the dominant EEG rhytham during anesthesia in the Java monkey: correlation with anesthetic potency. Anesthesiology. 1977;46(4):252-9.

27. Lewis LD, Weiner VS, Mukamel EA, Donoghue JA, Eskandar EN, Madsen JR, et al. Rapid fragmentation of neuronal networks at the onset of propofolinduced unconsciousness. Proc Natl Acad Sci. 2012;109:E3377-86.

28. Devor M, Zalkind V. Reversible analgesia, atonia, and loss of consciousness on bilateral intracerebral microinjection of pentobarbital. Pain. 2001;94:10112 .

29. Lee JM, Akeju O, Terzakis K, Pavone KJ, Deng H, Houle TT, et al. A prospective study of age-dependent changes in Propofol-induced electroencephalogram oscillations in children. Anesthesiology. 2017;127: 293-306.

30. Choi B-M. Characteristics of electroencephalogram signatures in sedated patients induced by various anesthetic agents. J Dent Anesth Pain Med. 2017:17:241-51.

31. Messner M, Beese U, Romstöck J, Dinkel M, Tschaikowsky K. The bispectral index declines during neuromuscular block in fully awake persons. Anesth Analg. 2003;97:488-91.

32. Shin YC, Kim Sl, Ok SY, Kim SC. The influence of a muscle relaxant on bispectral index during the propofol induction of anesthesia. Korean $J$ Anesthesiol. 2008;54:373-7.

33. Bruhn J, Bouillon TW, Shafer SL. Electromyographic activity falsely elevates the bispectral index. Anesthesiology. 2000;92:1485-7.

\section{Publisher's Note}

Springer Nature remains neutral with regard to jurisdictional claims in published maps and institutional affiliations.

Ready to submit your research? Choose BMC and benefit from:

- fast, convenient online submission

- thorough peer review by experienced researchers in your field

- rapid publication on acceptance

- support for research data, including large and complex data types

- gold Open Access which fosters wider collaboration and increased citations

- maximum visibility for your research: over $100 \mathrm{M}$ website views per year

At BMC, research is always in progress.

Learn more biomedcentral.com/submissions 